unacceptable disrespect for early human life. Perhaps a distinction has to be drawn between deliberately risking the induction of multiple pregnancy with the full intention of using fetal reduction and resorting to fetal reduction only when high order multiple pregnancy occurs inadvertently.

There is no information on the emotional consequences of selective reduction on the mother or the surviving children. In a short time mothers go from the emotional problems of prolonged infertility to high order multiple pregnancy and fetal reduction. Initially, they may experience relief after selective reduction, but they may later have feelings of guilt or bereavement. Careful follow up is required.

Fetal reduction is an example of technical advance moving ahead of public opinion, and open discussion of the obstetric, legal, and ethical issues is required before the practice can be fully accepted. Clearly, prevention is better than cure, and everything should be done to prevent the dilemma for parents in the first place. It may well come to be recognised, however, that selective fetal reduction is the humane option for some couples faced with the horrifying potential consequences of multiple births.

Professor of Obstetrics and Gynaecology,

Ninewells Hospital and Medical School,

Dundee DD1 9SY

1 Botting BJ, Davies IM, Macfarlane EJ. Recent trends in the incidence of multiple births and associated mortality. Arch Dis Child 1987;62:941-50.

Walker EM, Patel NB. Mortality and morbidity in infants born between 20 and 28 weeks gestation. Br J Obstet Gynaecol 1987;94:670-4.

3 Hobbins JC. Selective reduction-a perinatal necessity? N Engl f Med 1988;318:1062-3.

4 Levene MI. Grand multiple pregnancies and demand for neonatal intensive care. Lance 1986;ii:347-8.

Kanhai HHH, Van Rijssel EJC, Meerman RJ, Gravenhorst JB. Selective termination in quintuple pregnancy during first trimester. Lancet 1986;i:1447.

Kerenyi TD, Chitkara U. Selective birth in twin pregnancy with discordancy for Down's syndrome. $N$ Engl f Med 1981;304:1525-7.

Berkowitz RL, Lynch L, Chitkara U, et al. Selective reduction of multifetal pregnancies in the firs trimester. N Engl f Med 1988;318:1043-7.

8 Voluntary Licensing Authority for Human In Vitro Fertilisation and Embryology. Third report. London: Voluntary Licensing Authority, 1988. (Available from the Secretariat, 20 Park Crescent, London WIN 4AL.

9 Evans MI, Fletcher JC, Zador IE, Newton BW, Quigg MH, Struyk CD. Selective first-trimester termination in octuplet and quadruplet pregnancies: clinical and ethical issues. Obstet Gynecol 1988;71:289-96.

\title{
Intra-arterial hepatic chemotherapy for liver malignancy
}

\author{
Not yet proved to prolong survival
}

The rationale for giving patients with tumours of the liver intra-arterial hepatic chemotherapy is anatomical, pharmacological, and toxicological. The blood supply to liver tumours derives mainly from the hepatic arterial bed, and consequently more drug should reach the tumour if given by this route rather than either systemically or into the portal circulation. ${ }^{1-3}$ Using drugs with short plasma half lives that can be efficiently extracted by the liver should diminish systemic toxicity, and the ability to deliver high local concentrations of drug may increase the regression of tumours. ${ }^{4}$ Giving drugs by infusion and decreasing hepatic arterial flow may further enhance toxicity. ${ }^{5}$ Concomitant injection of inert particulate matter-for example, biodegradable starch microspheres-may not only decrease blood flow but also infarct the tumour. ${ }^{6} \mathrm{~A}$ recent advance has been to show the prolonged retention in sites of metastatic tumour of lipophilic cytotoxic drugs injected in lipid contrast medium.

Numerous non-randomised studies of intra-arterial hepatic chemotherapy in primary and secondary liver cancer have been conducted since the technique was described in the early $1950 \mathrm{~s} .{ }^{89}$ Higher rates of response than for systemic chemotherapy have been seen in patients with hepatoma, ${ }^{10}$ metastatic ocular melanoma, ${ }^{11}$ and metastatic colorectal carcinoma. ${ }^{12}$ But randomised studies are needed to determine toxicity, rates of response, and the benefits to survival. So far randomised studies have been reported only for metastatic colorectal carcinoma.

The California Oncology Group compared systemic and intra-arterial fluorouracil in 61 patients with liver metastases of colorectal carcinoma and found no difference in rate of response or survival. ${ }^{13}$ Two separate trials-from the Memorial Sloan Kettering Cancer Research Centre and the Northern California Oncology Group—have used floxuridine and shown a higher rate of response (50-59\%) with intraarterial hepatic chemotherapy than with systemic chemotherapy $(10-17 \%){ }^{14}$ is Patients failing to respond to systemic chemotherapy were crossed over to intra-arterial hepatic chemotherapy, and up to a quarter responded further. The crossover made true assessment of survival impossible, but this was not the primary aim of the trials. In the trial at the Sloan Kettering the median survival of the patients starting treatment with intra-arterial hepatic chemotherapy was 17 months compared with 12 months for those starting treatment with systemic chemotherapy. This difference was not significant but may show some survival benefit in certain subgroups of patients-for example, those not prone to developing extrahepatic disease. The main toxicity of intraarterial hepatic chemotherapy was chemical hepatitis, biliary sclerosis, and gastroduodenal ulceration. Some patients had liver pain, but abdominal pain was more common and was caused by misperfusion of the gastrointestinal tract. Indeed, systemic infusional chemotherapy was associated with greater toxicity, especially diarrhoea, and the overall quality of life of patients given intra-arterial hepatic chemotherapy was judged to be better than that of those given systemic chemotherapy. ${ }^{14}$ An important problem, however, has been the higher rate of extrahepatic metastases in those given intra-arterial hepatic chemotherapy.

The morbidity caused by intra-arterial hepatic chemotherapy has decreased with experience of the technique. ${ }^{16}$ Ambulatory patients may be treated with internally or externally placed pumps. ${ }^{17}$ One important factor limiting the use of intra-arterial hepatic chemotherapy is the lack of effective drugs to treat tumours such as hepatoma and colorectal carcinoma; other factors include cost and the continued growth of disease in other sites.

Clinical Research Fellow,

S T A MALIK

Imperial Cancer Research Fund,

London WC2A 3PX

Consultant Oncologist,

Imperial Cancer Research Fund,

Department of Medical Oncology,

St Bartholomew's Hospital,

London EC1A 4BE

1 Breedis C, Young C. The blood supply of neoplasms of the liver. Am $\mathcal{A}$ Pathol 1954;30:969-74. 2 Ridge JA, Bading JR, Gelbard AS, Benua RS, Daly JM. Perfusion of colorectal hepatic metastases: relative distribution of flow from the hepatic artery and portal vein. Cancer 1987;59:1547-53. 
3 Siguardson ER, Ridge JA, Kemeny N, Daly JM. Tumor and liver drug uptake following hepatic artery and portal vein infusion. 7 Clin Oncol 1987;5:1836-9.

Chen HSG, Gross JF. Intra-arterial infusion of anti-cancer drug: theoretic aspects of drug delivery and review of responses. Cancer Treat Rep 1980;64:31-40.

5 Lokich J, Eisenmerger W. Ambulatory pump infusion devices for hepatic artery infusion. Semin Oncol 1983;10:183-90.

6 Dakhil S, Eisenmenger W, Cho K, Niederhuber J, Doan K, Wheeler R. Improved regional selectivity of hepatic arterial bischlorethylnitrosourea with degradable microspheres. Cancer $1982 ; 50: 631-5$

7 Konno T, Meada H, Iwai K, Maki S, Tashiro S, Uchida M, Migauchi Y. Selective targeting of anticancer drug and simultaneous image enhancement of solid tumors by arterially administered lipid contrast medium. Cancer 1984;54:2367-74.

8 Klopp CT, Bateman J, Berry N, Alford C, Winship T. Fractionated regional cancer chemotherapy Cancer Res 1950;10:229.

9 Bierman HR, Byron RL, Miller ER, Shimkin MB. Effects of intra-arterial administration of nitrogen mustard. Am f Med 1950;8:535.

10 Bern MM, McDermott W, Cady B. Intra-arterial hepatic infusion and intravenous adriamycin for the treatment of hepatollular carcinoma Cancer 1978:42:399-405.

11 Mavligit G, Carrasco N, Papadopoulos N, Charnsangvei C, Wallace S. Regression of ocular melanoma metastatic to the liver after chemoembolisation with cisplatinum and polyvinyl sponge. Proceedings of the American Society for Clinical Oncology 1987;6:210.

12 Ensminger N, Niederhuber J, Gyves J. Effective control of liver metastases from colon cancer with an implanted system for hepatic arterial chemotherapy. Proceedings of the American Society for Clinical Oncology 1982;1:94.

13 Grage TB, Vassillopoulos PP, Shingleton WW. Results of a prospective randomised study of hepatic artery infusion with 5-fluorouracil versus intravenous 5 -fluorouracil in patients with hepatic metastases. A Central Oncology Group study. Surgery 1979;86:550-5.

14 Kemeny N, Daly J, Reichman B, Geller N, Botet J, Oderman P. Intrahepatic or systemic infusion of fluorodeoxyuridine in patients with liver metastases from colorectal carcinoma: a randomised trial. Ann Intern Med 1987;107:459-65.

15 Hohn D, Stagg R, Friedman M, et al. The NCOG randomised trial of intravenous versus intrahepatic FUDR for colorectal cancer metastatic to the liver. Proceedings of the America Society for Clinical Oncology 1987;6:85.

16 Oberfield RA. Intra-arterial hepatic infusion chemotherapy in metastatic liver cancer. Semin Oncol 1983;10:206-14.

17 Lokich J, Ahlgren J, Gullo J. A randomised trial of standard bolus 5-fluorouracil versus protracted infusional 5-FU in advanced colon cancer. Proceedings of the American Society for Clinical Oncology 1987;6:81.

\title{
Depression resistant to tricyclic antidepressants
}

\author{
Adding lithium will often work
}

Up to a third of patients with major depression either fail to respond or respond only partially to treatment with tricyclic antidepressants. ${ }^{1}$ In some cases specific psychotherapeutic or social intervention will be required, but further drug treatment will be considered for many. What are the possibilities?

An initial step is to see whether the dose of the tricyclic antidepressant may be increased. The proportion of patients responding increases as the dose is raised, and many patients may be able to tolerate more than the usual $150 \mathrm{mg}$ daily. ${ }^{2}$ Whether monitoring plasma concentrations of tricyclic antidepressants can help determine correct dosage is disputed, ${ }^{34}$ and the usual course is to increase the dose until definite but tolerable side effects are apparent. ${ }^{5}$

For patients with severe depression, when endogenous symptoms are prominent, the usual practice when treatment with tricyclic antidepressants is ineffective is to consider electroconvulsive therapy. At least half of this group of patients will improve after electroconvulsive therapy. ${ }^{67}$ Subjects who are not responding to unilateral electroconvulsive therapy should have a trial of bilateral treatment before electroconvulsive therapy is abandoned. ${ }^{8}$ Electroconvulsive therapy is particularly useful in patients with psychotic depression, but in such patients combining tricyclic antidepressants with neuroleptic drugs may yield rates of response similar to those with electroconvulsive therapy (about $80 \%)^{9}$

Electroconvulsive therapy usually necessitates admission to hospital, and many patients are reluctant to receive it. There is thus much interest in alternative drug treatments, and recent attention has focused on the use of lithium. Two controlled investigations have suggested that adding lithium to tricyclic antidepressants in patients who have not responded produces a clear improvement in about $50-60 \% .^{111}$ Although some may respond within two to three days, the more usual pattern is for a gradual remission over about three weeks while lithium concentrations are maintained between $0.5-0.8 \mathrm{mmol} / 1 .{ }^{12}$ Whether this response is attributable to an antidepressant action of lithium alone ${ }^{13}$ or to a synergism between lithium and the tricyclic antidepressant is not clear. ${ }^{11} 14$

Ineffective treatment with tricyclic antidepressants may also be supplemented by a small daily dose of liothyronine (triiodothyronine) $(20-40 \mu \mathrm{g})$. Both anecdotal reports and one controlled trial suggest that $60-70 \%$ of patients may show some improvement within a few days. ${ }^{15}$ Recent assessments have, however, been less encouraging, ${ }^{16}{ }^{17}$ and adding lithium is probably a more effective strategy. ${ }^{17}$

If a patient has been able to tolerate a full dose of a tricyclic antidepressant with no improvement changing to another tricyclic antidepressant or a newer antidepressant will probably not be worth while. If combined treatment with lithium and tricyclic antidepressants has been ineffective a monoamine oxidase inhibitor should be considered. Given in high enough doses monoamine oxidase inhibitors are effective antidepressants, ${ }^{18}$ and they often help in patients who have failed to respond to other antidepressants and electroconvulsive therapy. ${ }^{18} 19$ It was originally suggested that depressed patients who responded to monoamine oxidase inhibitors were more likely to have an atypical presentation of depression, ${ }^{20}$ and the results of some, but not all, recent investigations have supported this proposal. ${ }^{1821}$ The balance of evidence shows that patients whose depressive disorder is characterised by mood reactivity, increased sleep and appetite, and feelings of anxiety and anger will show a good rate of response (about $70 \%$ ) to monoamine oxidase inhibitors. ${ }^{22}$ Clinical impression is that such patients are unlikely to be helped by electroconvulsive therapy. ${ }^{5}$

Lithium may also potentiate treatment with monoamine oxidase inhibitors, and there are reports that lithium and tranylcypromine may relieve severe depressive states that have not responded to lithium and a tricyclic antidepressant combined or to a monoamine oxidase inhibitor given alone. ${ }^{23}$ Some believe that adding tryptophan to lithium and a monoamine oxidase inhibitor may help in patients with particularly resistant depression, and about half of this group of patients may show substantial improvement with this combination. ${ }^{24}$ Good clinical effects have also been reported with combining lithium, tryptophan, and clomipramine. ${ }^{25}$

The drugs considered most effective in resistant depression are characterised by their ability to produce a striking enhancement in some aspects of brain serotonin function..$^{1423}$ There has been much progress recently in identifying specific subtypes of brain serotonin receptors ${ }^{26}$ and selective drugs for these receptors are becoming available. Whether such drugs have useful antidepressant properties will therefore be of both clinical and scientific interest.

P J COWEN

Medical Research Council Clinical Scientist,

Medical Research Council Unit of Clinical Pharmacology,

Littlemore Hospital,

Oxford OX4 4XN 